\title{
Diverse Synaptic Plasticity Induced by the Interplay of Ionic Polarization and Doping at Salt-Doped Electrolyte/Semiconducting Polymer Interface
}

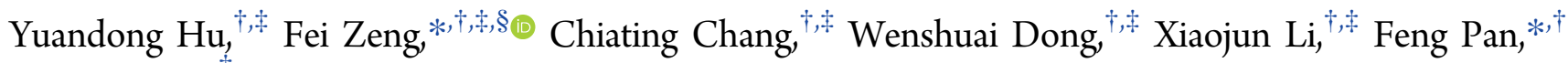 \\ and Guoqi $\mathrm{Li}^{\ddagger}$
}

${ }^{\dagger}$ Laboratory of Advanced Materials (MOE), School of Materials Science and Engineering, and ${ }^{\ddagger}$ Center for Brain Inspired Computing Research (CBICR), Tsinghua University, Beijing 100084, People's Republic of China

${ }^{\S}$ Key Laboratory of Microelectronic Devices \& Integrated Technology, Institute of Microelectronics, Chinese Academy of Sciences, Beijing 100029, People's Republic of China

\section{Supporting Information}

ABSTRACT: $\mathrm{Pt} / \mathrm{Ca}^{2+}$-polyethylene oxide/polymer poly[3-hexylthiophene2,5-diyl]/Pt devices were fabricated, and their pulse responses were studied. The discharging peak, represented by the postsynaptic current (PSC), first increases and then decreases with increasing input number in a pulse train. The weight of the PSC decreased for low-frequency stimulations but increased for high-frequency stimulations. However, the peak of the negative differential resistance during the charging process varied following the opposite trend. These behaviors suggested the ability for transferring the signal bidirectionally, confirming the equivalence between the ionic kinetics of our device and the transmitter kinetics of one kind of synapse. A facilitation $(F)$-depression $(D)$ interplay model corresponding to the ionic polarization and doping interplay at the electrolyte/semiconducting polymer interface was adopted to successfully mimic the weight modification of the PSC. The simulation results showed that the observed synaptic plasticity was caused by the great disparity between the recovery time constants of $F$ and $D\left(\tau_{F}\right.$ and $\left.\tau_{D}\right)$. Moreover, such an interplay could inspire the features of responses to post-tetanic stimulations. Our study suggested a means to realize synaptic computation.

\section{INTRODUCTION}

Until now, a lot of efforts have focused on finding artificial materials to realize the functions of synapses and neurons, ${ }^{1-4}$ which could be used as elements to create neuromorphic networks and brainlike computations. ${ }^{5}$ Such endeavors started long ago to explore the mysteries of signal handling and memory of the neural system. ${ }^{6,7}$ Spikes resembling action potentials were generated, followed by electrical injection using polyelectrolyte membranes without lipids. ${ }^{6}$ Lipid membranes acting as a carrier for signal propagation have also been studied thoroughly. ${ }^{7}$ Recently, many studies have found that systems containing salt-doped organic electrolyte/semiconducting polymers behave rather similar to biological synapses. Ionic migration at the interface plays a critical role in modulating the signal strength and plasticity. In particular, frequency selectivity was first discovered in $\mathrm{Pt} /$ polyethylene oxide $(\mathrm{PEO})+\mathrm{Li}^{+}$/ poly(3-hexylthiophene-2,5-diyl) (P3HT)/Pt device, for which the system responded in depression at a low-frequency stimulation (LFS) but responded in potentiation at a highfrequency stimulation (HFS)., ${ }^{8,9}$ When the semiconducting layer was changed to modulate the de-doping rate of ions, the frequency selectivity became long-term reserved, exhibiting spike-rate-dependent plasticity (SRDP). ${ }^{10}$ Moreover, the threshold of depression-potentiation in the frequency selectivity exhibited a sliding behavior ${ }^{11}$ which is consistent with the BCM (Bienenstock, Cooper, and Munro) learning rule to solve the problem of stability and adaptivity. ${ }^{12,13}$ In addition, the monotonous plasticity or the simple Hebbian learning rule could simply be simulated by the pulse response of a salt-doped electrolyte. ${ }^{14}$ Therefore, these studies suggest that the ionic kinetics in salt-doped electrolyte/semiconducting polymer systems could act as prototypes for simulating the ionic flux and transmitter release of neural systems. It is important for us to recognize the intrinsic mechanism of such ionic kinetics.

As known from neuroscience, short-term plasticity (STP) is regarded to be critical in signal handling. ${ }^{15,16}$ The messengers, also known as neural transmitters, are subjected to the interplay between facilitation and refractory depression effects, which is the stem of diversity of plasticity. ${ }^{15,17-19}$ The former is caused by the calcium-dependent release probability, and the latter is the result of release sites becoming ineffective after release. The microscopic mechanisms and kinetics of facilitation and

Received: November 13, 2016

Accepted: February 14, 2017

Published: February 28, 2017 
(a)

(b)
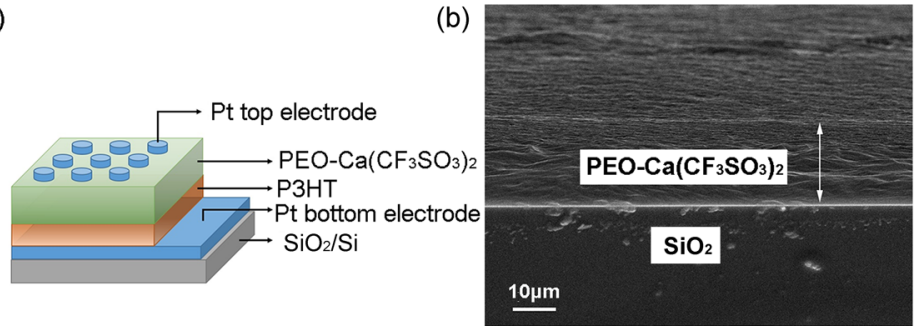

(c)

(d)
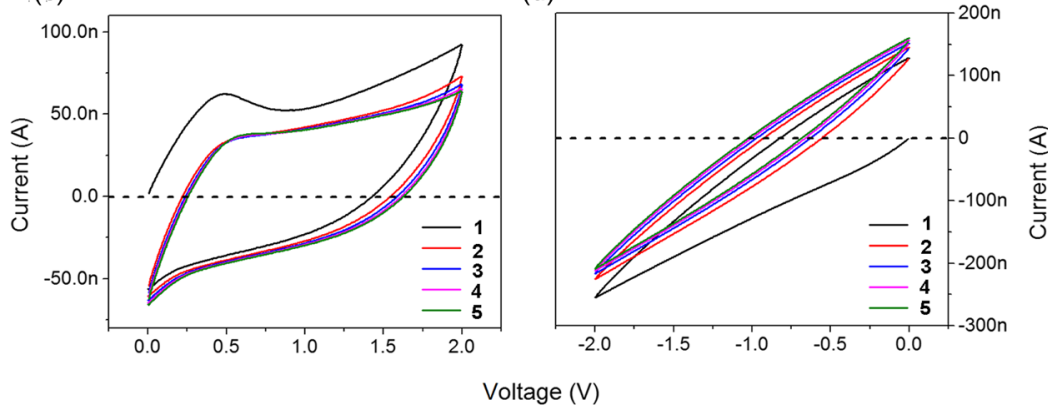

Figure 1. Schematic of the $\mathrm{Pt} / \mathrm{PEO}-\mathrm{Ca}\left(\mathrm{CF}_{3} \mathrm{SO}_{3}\right)_{2} / \mathrm{P} 3 \mathrm{HT} / \mathrm{Pt}$ device and its direct current-voltage (dc $\left.I-V\right)$ characteristics. (a) Device configuration. (b) SEM cross-sectional image of the device, showing that the thickness of the PEO complex is approximately $20 \mu \mathrm{m}$. (c,d) $I-V$ properties obtained by using a sweeping prototype at a rate of $100 \mathrm{~V} / \mathrm{s}$ in the voltage range of (c) $0-2 \mathrm{~V}$ and (d) 0 to $-2 \mathrm{~V}$. Five sweeping cycles were applied.

depression could vary because of the variety of structures of neurons and synapses. With the models available, the physicochemical mechanism to simulate the corresponding plasticity can be found. For example, a salt-doped electrolyte device could be used to simulate a monotonous plasticity (potentiation or depression), in which the transmitter release ability is either high or low, ${ }^{16,17,20,21}$ and the recovery of the electric natural region or the enhanced ionic polar region corresponds to either a facilitation $(F)$ or a depression $(D)$ process. ${ }^{14}$ However, it is hard to use such a simple structural model to simulate a more complex plasticity such as frequency selectivity because neither $F$ nor $D$ can predominate over the whole period of external stimulation.

The introduction of an electrolyte/semiconductor interface plays an important role in modulating the ionic kinetics and provides an opportunity to simulate a more complex plasticity. In these devices, ionic doping into the semiconductor layer occurs during pulse stimulation, and de-doping occurs in the interval between two pulses, which overlap with each other both temporally and spatially. The frequency selectivity in $\mathrm{Pt} /$ $\mathrm{Li}^{+}-\mathrm{PEO} / \mathrm{P} 3 \mathrm{HT} / \mathrm{Pt}$ devices can be explained from the phenology point of view, ${ }^{8}$ and a structural model can be established to demonstrate the mechanism of SRDP., ${ }^{9,10}$ Because the modifications of the structure or the conformation at the electrolyte/semiconductor interface cannot be obtained during pulse stimulation, only the ionic kinetics of the saltdoped electrolyte and the corresponding pulse responses can be simulated on the level of molecular dynamics. ${ }^{22}$ However, interactions between ionic accumulation and doping at the interface naturally exist in both the temporal and spatial scale, which should be the critical factors for frequency selectivity generation. This leads us to consider the kinetic model of the interplay between $F$ and $D$ known in neuroscience and the contribution of the interplay to the pulse responses. ${ }^{17}$

In this work, we studied the pulse responses and plasticity of $\mathrm{Pt} / \mathrm{Ca}\left(\mathrm{CF}_{3} \mathrm{SO}_{3}\right)_{2}$-doped $\mathrm{PEO} / \mathrm{P} 3 \mathrm{HT} / \mathrm{Pt}$ devices, finding that they behaved equivalently to the Schaffer collateral (SC) synapses of rat brains. ${ }^{17}$ Thus, the interplay model of $F$ and $D$ in neuroscience was adopted to simulate weight modifications, ${ }^{17}$ in which the $F$ and $D$ components would correspond to the increase and decrease of $\mathrm{Ca}^{2+}$ ions accumulated or ionic polarization and doping at the $\mathrm{PEO} / \mathrm{P} 3 \mathrm{HT}$ interface. In addition, we demonstrated that the model can be extended to other complex plasticities, for example, weight modification due to post-tetanic stimulation.

\section{RESULTS AND DISCUSSION}

2.1. Electrical Properties and Facilitation-Depression Combined Short-Term Plasticity. Figure $1 \mathrm{a}, \mathrm{b}$ shows the device structure with $\mathrm{EO} / \mathrm{Ca}^{2+}$ molar ratio of $32: 1$, and Figure $1 \mathrm{c}, \mathrm{d}$ shows direct current-voltage ( $\mathrm{dc} I-V)$ characteristics. The structural results from the X-ray diffraction (XRD) spectra indicate that the P3HT crystallites were not detected, and the electrolyte consisted of the crystallized PEO and the amorphous $\mathrm{PEO}-$ salt complex in the $\mathrm{PEO}-\mathrm{Ca}^{2+} / \mathrm{P} 3 \mathrm{HT}$ $\left(\mathrm{EO} / \mathrm{Ca}^{2+}=32: 1\right)$ device (Figure S1). The $\mathrm{Ca}^{2+}$ transport channels mainly existed in the amorphous region, which was surrounded by crystalline lamellae. The Raman results indicate that the initial state of P3HT is not doped by the salt (Figure S2) but might enhance the diffusion probability of ions into the P3HT layer.

The sweeping rate used to measure the $I-V$ characteristics was $100 \mathrm{~V} / \mathrm{s}$. Hysteresis loops appeared because of ion migration and the formation of an electric double layer of the PEO electrolyte. When a P3HT layer was introduced, the $I-V$ characteristics showed a rectification behavior and a negative differential resistance (NDR) effect under a positive bias, as shown in Figure 1c. Because the $I-V$ curves of the PEO complex single-layer device exhibited a symmetric shape and no NDR effect (Figure S3), this phenomenon is attributed to the p-type P3HT, owing to its $\pi$-electron-rich property. When a positive bias was applied, the electrostatic induction of $\pi$ - 
(a)

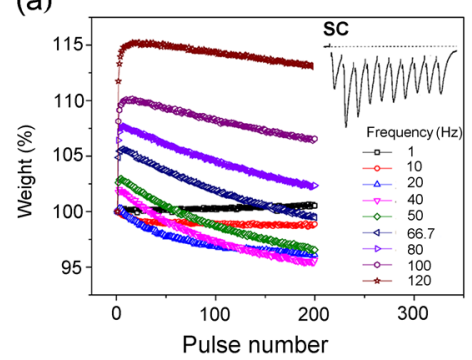

(b)
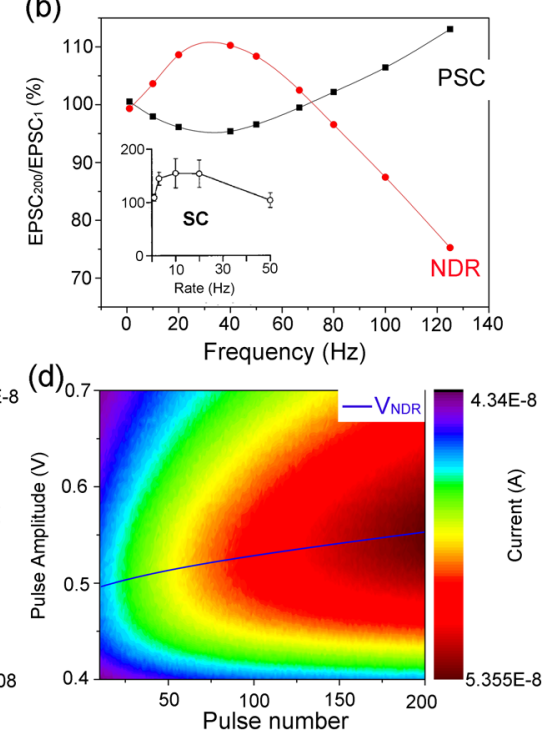

Figure 2. Variations in the pulse responses with pulse number and frequency. The train of triangular pulses was added on the device with a pulse amplitude of $0.7 \mathrm{~V}$, a loading rate of $100 \mathrm{~V} / \mathrm{s}$, and a pulse number of 200 . (a) Weight variations with the pulse number at different frequencies. Inset: PSC of the SC synapse of rat brain varying with input number. ${ }^{21}$ (b) Weight variations with frequency. The weight values were calculated by using the data obtained from the responses to the last pulse. The black-square line represents the weight of the discharging peaks, whereas the red-square line represents that of the NDR peaks. Inset: Weight modifications of the SC synapse vs frequency. (c,d) Variation in the sections identified during the charging processes $(0.4-0.7 \mathrm{~V}$ ) with the pulse number (from 10 to 190) under (c) $40 \mathrm{~Hz}$ and (d) $80 \mathrm{~Hz}$ stimulations, respectively. The black lines represent the trend of the NDR voltage $\left(V_{\mathrm{NDR}}\right)$ with the pulse number. The color maps represent the current values at the NDR peaks.

electrons appeared because of an accumulation of $\mathrm{Ca}^{2+}$ ions at the PEO/P3HT interface. ${ }^{23}$ This generated an additional electric field that produced a reduction in the offset potential (Figure 1b), which caused an evident decrease in the current and an NDR effect. However, when a negative bias was applied, the $\pi$-electrons of P3HT could not cause significant polarization with the $\mathrm{CF}_{3} \mathrm{SO}_{3}{ }^{-}$ions. Thus, the migration of ions hardly meets any obstacles, and no NDR effect appeared. Besides, the current values in the negative sweeping direction are higher than those in the positive sweeping direction, suggesting a rectification effect in the device. We also examined the resistance of the P3HT film (Figure S4) and confirmed that it contributed less to the whole resistance value and that the interface effect is the origin of the rectification, which could be reflected in the impedance spectra (Figure S5).

To further investigate the response of these devices, we looked at the response to trains of triangular pulses with an amplitude of $0.7 \mathrm{~V}$, a loading rate of $100 \mathrm{~V} / \mathrm{s}$, a pulse number of 200 , and a frequency range of $1-125 \mathrm{~Hz}$. The discharging peak after each pulse can be identified as the postsynaptic current (PSC) adopted in the neuroscience. ${ }^{8,10,13,24}$ The ratio between the Nth pulse and the first pulse in a series of PSCs was defined as the synaptic weight $\left(W=\mathrm{PSC}_{N} / \mathrm{PSC}_{1}\right)$. The variation of $W$ with the input number at different frequencies is shown in Figure 2a. In our experiment, the weight modification at $1 \mathrm{~Hz}$ was set as baseline ( $W=100 \%)$ because the PSC did not vary at that frequency. As shown in Figure $2 \mathrm{a}$, when the input frequency was between 10 and $20 \mathrm{~Hz}$, the weight of the PSC decreased to a stable value with a time constant of seconds. When the input frequency was increased further to relatively high values (approximately $50 \mathrm{~Hz}$ ), the PSC showed a discernible increase in the first several pulses and then decreased, showing a synaptic weight lower than $100 \%$ at the end of the pulse train. When the input frequency was very high, that is, $125 \mathrm{~Hz}$, the PSC rose to a high value, with a weight of
$116 \%$ (facilitation period) and then gradually decreased to a value of approximately $113 \%$ (depression period). Such a facilitation-depression transition is equivalent to the shortterm plasticity of SC synapses of the rat brain (inset in Figure $2 a)$, combining both facilitation and depression on a different time scale. ${ }^{17}$

The weight modification with respect to the input frequency is plotted in Figure $2 \mathrm{~b}$ by using the PSC values of the 200th pulse responses (black-dot line). These results show that the PSC facilitated at high rates but depressed during lower stimulation rates. Moreover, the $W-f$ function of the $\mathrm{Ca}-$ $\mathrm{PEO} / \mathrm{P} 3 \mathrm{HT}$ device is consistent with those of $\mathrm{Li}-\mathrm{PEO} / \mathrm{P} 3 \mathrm{HT}$ and $\mathrm{Mg}-\mathrm{PEO} / \mathrm{P} 3 \mathrm{HT}$ devices, which depressed at LFS and facilitated at HFS. ${ }^{8,25,26}$ Such frequency selectivity was first proposed by the CLO (Cooper, Liberman, and Oja) learning rule to provide a selective response to various signals, ${ }^{12,27}$ which shows that the difference among ion-molecule interactions should be intrinsic, supporting signal selectivity and computation. ${ }^{15}$ The composition of EO/ $\mathrm{Ca}^{2+}=32: 1$ that we chose is a conventional ratio, as we used in our previous studies. Here, we have examined the XRD patterns for the samples with $\mathrm{EO} / \mathrm{Ca}^{2+}$ $=32: 1$ and 16:1 in Figure S1. We found that when EO/ $\mathrm{Ca}^{2+}$ is larger than 24:1, the device would exhibit frequency selectivity; otherwise, the synaptic weight could only monotonically increase with the frequency. All electrical experiments were performed at room temperature. The ionic conductivity of the PEO-salt complex will significantly decrease as the temperature drops. However, when the temperature rises, the PEO layer may turn to a viscous state.

According to the results of doping several types of ions, we find that usually the depression values under low frequencies are moderate around $1-10 \%$, but the potentiation values under high frequencies varied greatly with the type of ions up to $150 \%{ }^{8-10,26}$ Apparently, the type of ions is one of the most important factors influencing the value of weight modification. 
The atomic mass of $\mathrm{Ca}$ ion is larger than those of $\mathrm{Li}$ and $\mathrm{Mg}$ ions so that the migration rate of $\mathrm{Ca}^{2+}$ is lower than those of $\mathrm{Li}^{+}$ and $\mathrm{Mg}^{2+8,26}$ This may be a reason that the absolute weight values are low in this work. Considering that a synapse system comprises several types of ions, we guess that the difference in the weight value induced by the type of ions could be one element for computing in a neuromorphic network. Therefore, if we need a large value of weight modification, we could choose the ions with a small atomic mass. We are finding methods to distinguish factors influencing the weight value quantitatively, especially solve the relationship between the weight value and the ion mass.

The charging process was also considered, showing that the largest charging current, that is, the value of the NDR peak, decreased first at low input rates and then increased at higher input rates, which is in contrast to the PSC behavior during the discharging process. This could be seen when the weight modifications of the NDR peaks versus frequency were calculated, as shown by the red-dot line in Figure $2 \mathrm{~b}$. Such plasticity of the charging process can be demonstrated in detail by using the pulse responses to stimulations at 40 and $80 \mathrm{~Hz}$. The current of the charging process $(0.4-0.7 \mathrm{~V})$ during each pulse in the depression stage $(N=10-190)$ is shown in Figure $2 \mathrm{c}$,d. As the number of pulses increases, the current and bias of the NDR peak also increase, suggesting a nonlinear variation in the electrical resistance, similar to the one seen in memristor devices. $^{2,28,29}$ The weight represented by the NDR peak leads to an opposite selectivity of pulse frequency, shown by the red dots in Figure $2 \mathrm{~b}$, and coincides with the $W-f$ variation of SC synapses (inset in Figure 2b). This phenomenon has also been observed in $\mathrm{Mg}-\mathrm{PEO} / \mathrm{P} 3 \mathrm{HT}$ devices. ${ }^{26}$ Thus, the system displays the potential for bidirectional signal transfer. This feature is not attended in either organic electronics or ionics and is only observed in the system doped by the binary valence ions according to our recent studies. ${ }^{8,10,14,25,26,29}$ Therefore, these results suggest that the size, valence state, and atomic mass of ions would become the gist of signal selectivity.

Recent studies reported mimicking SRDP by using memristors. $^{30-33}$ Compared with them, we are focusing on the role of ionic kinetics on the synaptic plasticity and similarity of the materials and device to the behavior of the biosynapse. Until now, the inorganic memristors exhibit a stronger potential in massive fabrication because of stability and a compatible technique with integration circuits. ${ }^{30,32,33}$ The weight variations in the charging and discharging process suggest that our system is usable for bidirectional interplay and signal transfer from the view of electronics. This is different from those studies where a synaptic device shows potentiation and depression under different voltage stimuli. ${ }^{30-33}$ Bidirectional transfer has been suggested early in Hebbian proposal. ${ }^{12,34}$ However, we are not sure that our results are consistent with those observed in biology and neuroscience, for example, back propagation from axon to dendrite. ${ }^{35}$ The physical mechanism in the phenomenon needs to be studied in depth. Anyway, it is possible to return the state of the post neuron at the same time sending out the signal using our system.

The results in Figure 2 also suggest that there should be a mechanism in the device that allows facilitation at the beginning but later depresses the pulse train (Figure 2a). Figure 3 illustrates the diverse plasticity and its intrinsic ionic kinetics. During the first several pulses, the polarization of ions under an external bias and the induction between $\mathrm{Ca}^{2+}$ ions and $\pi$-electrons in P3HT strengthened the internal electric field,

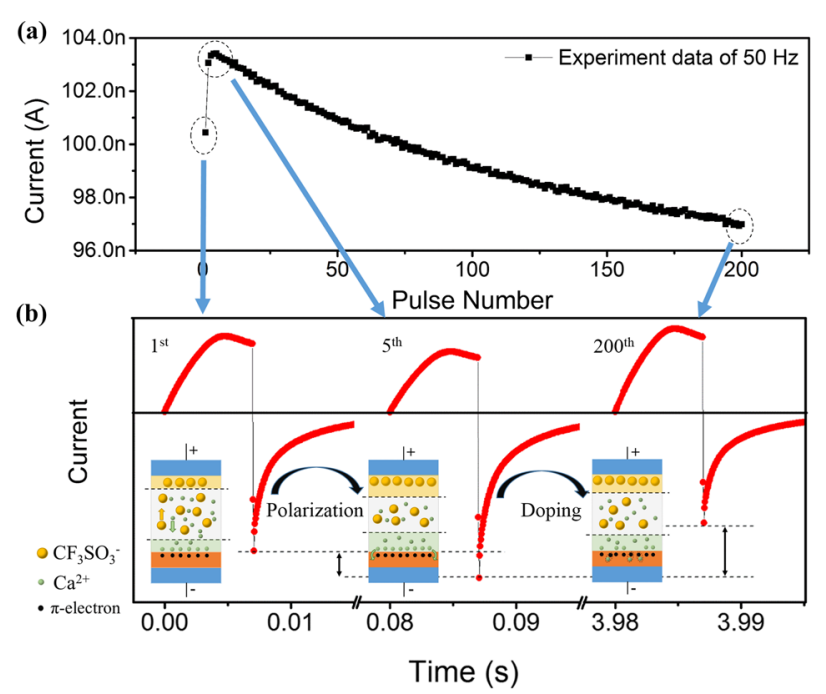

Figure 3. Internal ion dynamics at different periods of the pulse train. (a) PSC weight modification of $50 \mathrm{~Hz}$ pulses stimulation and (b) response current of the 1 st, 5 th, and 200th pulse. The insets show the ion distribution corresponding to each pulse. The discharging current peak increased by the polarization of ions when the 5th pulse loaded and decreased by the doping of $\mathrm{Ca}^{2+}$ when the 200th pulse loaded.

resulting in a fast facilitation process of a discharging current peak. After that, the weight decreased on a longer time scale, which could be attributed to the electrostatic-like doping process of the $\mathrm{Ca}^{2+}$ ions moving from the upper layer to the P3HT layer. The $\mathrm{Ca}^{2+}$ ions accumulated at the interface during the polarization process would be reduced by the electrostaticlike doping process, leading to a reduction in the interfacial inverse field and weakening the strength of the discharging peak. The doping process only occurs in the area under the island electrode, so that during the pulse intervals, locally doped $\mathrm{Ca}^{2+}$ ions decay and diffusion arises, as in the de-doping process. Usually, the electrochemical doping process between the $\mathrm{P} 3 \mathrm{HT}$ and $\mathrm{PEO}$ electrolytes is slower than the response time of polarization of the polymer dielectric, which is due to the interface barrier and the much slower ionic mobility in the semiconducting polymer compared to that of PEO. ${ }^{23,37}$ This is the reason the depression stage lasts much longer than the facilitation stage, as shown in Figure 2a. However, the PEO complex single-layer device simply showed facilitation plasticity under the same pulsed loads (section S2, Figure S6) because of the lack of doping from the upper layer to the P3HT layer.

Because the working currents are in the level of microamperes in most organic electronic experiments, we did not obtain the measurement platforms avoiding vibration. However, the pulse responses of our devices are more sensitive to the vibration because the current is lower than the level of $\mu \mathrm{A}$, so that we can perform the experiment in an open environment. In such case, both humidity and temperature influence the experimental results. We found previously that our samples could maintain selectivity at least for a week if only we placed them in the open environment during the experiment but failed after long-term exposure to air. ${ }^{10}$ The difference in the carrier migration ability of $\mathrm{PEO} / \mathrm{P} 3 \mathrm{HT}$ system in vacuum and in air was studied in a previous publication. ${ }^{36}$ The ionic conductivity of the PEO-based electrolyte was greatly increased upon hydration. Thus, when exposed to an open environment, ions had larger mobility to migrate from the PEO electrolyte layer to the semiconducting polymer layer. We 
suppose that operating in an open environment or higher humidity would strengthen the depression factor because the latter was attributed to the ionic doping process (discussed in Figure 3 and the following text). In the aspect of temperature, we suppose that a lower temperature will reduce the ionic mobility and doping level, and the depression factor would be weakened. The situation would be complicated if the temperature was enhanced. The heating effect of the current would induce local fusion of PEO. Both the influence of humidity and temperature needs to be verified in a platform precisely controlling the two factors.

The results in Figure 3 clearly demonstrate that the ionic polarization and doping interplay with each other at the interface, corresponding to the facilitation-depression interplay in neural synapses. ${ }^{17,38}$ This is the intrinsic reason for the analogies between the pulse response of our devices and SC synapses. A model in neuroscience has been developed to describe the incorporating mechanism of different short-term types of plasticity: depression, facilitation, and both depression and facilitation dominating synapses. The release value can be calculated as the product of the facilitation component $(F)$ and the depression component $(D)$, which are determined by the specific binding of release sites and residual presynaptic calcium. ${ }^{17}$ Both $F$ and $D$ decay for a constant time after the saltation, owing to an action potential, and finally stabilize at a constant value after several stimuli at a fixed frequency. Our previous study verified that the charging/discharging processes of the salt-doped PEO electrolyte correspond to the effective release sites of the transmitter and the $\mathrm{Ca}^{2+}$ flux in biological synapses if either $F$ or $D$ dominate. ${ }^{14}$ The key factor that influences the short-term plasticity is the elevation of residual presynaptic calcium $\left(\mathrm{Ca}_{\text {res }}\right)$. For all of these three different synapses, the release value is the product of the facilitation component $(F)$ and the depression component $(D)$, which are determined by the specific binding of the release sites and $\mathrm{Ca}_{\text {res. }}$. Thus, the kinetic model describing the $F-D$ interplay can be used to describe the pulse responses of our artificial devices, being able to simulate the short-term plasticity of two types of conventional synapses, that is, climbing fiber synapse (depression plasticity), parallel fiber synapse (facilitation plasticity), and SC synapse (facilitation and depression combined plasticity). ${ }^{14}$

The electrolyte/semiconductor interface differentiates this device from salt-doped electrolyte devices, and it is a critical element for frequency selectivity. Although the conformation or organization modification cannot be achieved while ions are passing through the interface, the system should be described using the facilitation-depression interplay modulated by the electrolyte/semiconductor interface. Considering the frequency selectivity, a similar but simpler quantitative model of $F$ and $D$ was adopted, referred to as temporal filtering. ${ }^{38-40}$ Using this model, the increase and decrease in the $\mathrm{Ca}^{2+}$ ions accumulated at the $\mathrm{PEO} / \mathrm{P} 3 \mathrm{HT}$ interface can be related to $F$ and $D$, respectively. Through the simulation process, we could subtract the kinetic parameters providing the base for the signal computation and extend the model to other types of complex short-term synaptic plasticity.

The amplitude of the PSC of each pulse is the product of several facilitation and depression components. The model we discuss here contains a single $F$ and a single $D$. Thus, the amplitude of the PSC, $A$, can be described as

$$
A=A_{0} F D
$$

where $F$ and $D$ correspond to the facilitation and depression components, respectively. The value of $F$ is larger than 1 , and the value of $D$ is smaller than 1 . After each pulse in a stimulation train, $F$ increases by a value $f$ and $D$ decreases by a value $d$.

$$
F \rightarrow F+f \quad D \rightarrow D-d
$$

Assuming that in a single test, $f$ and $d$ remain the same when the pulse number increases, both $F$ and $D$ converge exponentially back to 1 in the stimulation interval, expressed as

$$
\tau_{F} \frac{\mathrm{d} F}{\mathrm{~d} t}=1-F, \quad \tau_{D} \frac{\mathrm{d} D}{\mathrm{~d} t}=1-D
$$

Because of different time constants and varying patterns of $F$ and $D$, a single synapse can present both facilitation and depression on different time scales. In most cases, $\tau_{D}$ is larger than $\tau_{F}$, meaning that facilitation dominates in the early stage of a train of pulses and depression dominates in the later stage.

Under a positive bias, the internal electric field increases by the accumulation of $\mathrm{Ca}^{2+}$ ions at the interface and would weaken by the electrochemical doping of $\mathrm{Ca}^{2+}$ ions from PEO to P3HT. The larger internal field results in larger discharging currents and accumulation of $\mathrm{Ca}^{2+}$ ions, which operate as an electric double-layer capacitor and induce a facilitation component $F$. On the other hand, the dynamic doping process induces a depression component $D$. To explain the different plasticities corresponding to the different pulse intervals, we made a small adjustment in the above biological model, defining the PSC value as

$$
A_{N}=A_{0} F_{N} D_{N}
$$

where $A_{N}$ is the Nth PSC value, $A_{0}$ is the assumed baseline current, and $F_{N}$ and $D_{N}$ are the values of the $F$ and $D$ components after each stimulus, respectively. $F$ and $D$ evolve exponentially between two stimuli according to eq 3 , that is,

$$
\begin{aligned}
& F=1+\left(F_{N}-1\right) \cdot \alpha \cdot \exp \left(-\frac{\Delta t}{\tau_{F}}\right) \\
& D=1-\left(1-D_{N-1}\right) \cdot \beta \cdot \exp \left(-\frac{\Delta t}{\tau_{D}}\right) \\
& \Delta t=\frac{1}{r}-\lambda
\end{aligned}
$$

where $\alpha$ and $\beta$ are integration coefficients, $r$ is the pulse frequency, and $\lambda$ is the pulse width. Then, the recurrence relation between the components of the $N$ th pulse and the $(N$ - 1)th pulse can be defined as

$$
\begin{aligned}
& F_{N}=1+\left(F_{N-1}-1\right) \cdot a+f \\
& D_{N}=1-\left(1-D_{N-1}\right) \cdot b-d
\end{aligned}
$$

Here,

$$
a=\alpha \cdot \exp \left(-\frac{\Delta t}{\tau_{F}}\right) \quad b=\beta \cdot \exp \left(-\frac{\Delta t}{\tau_{D}}\right)
$$

Assuming $F_{0}=1$ and $D_{0}=1$ the general terms of $F_{N}, D_{N}$, and $A_{N}$ can be obtained as

$$
F_{N}=1+f \frac{1-a^{N}}{1-a}
$$



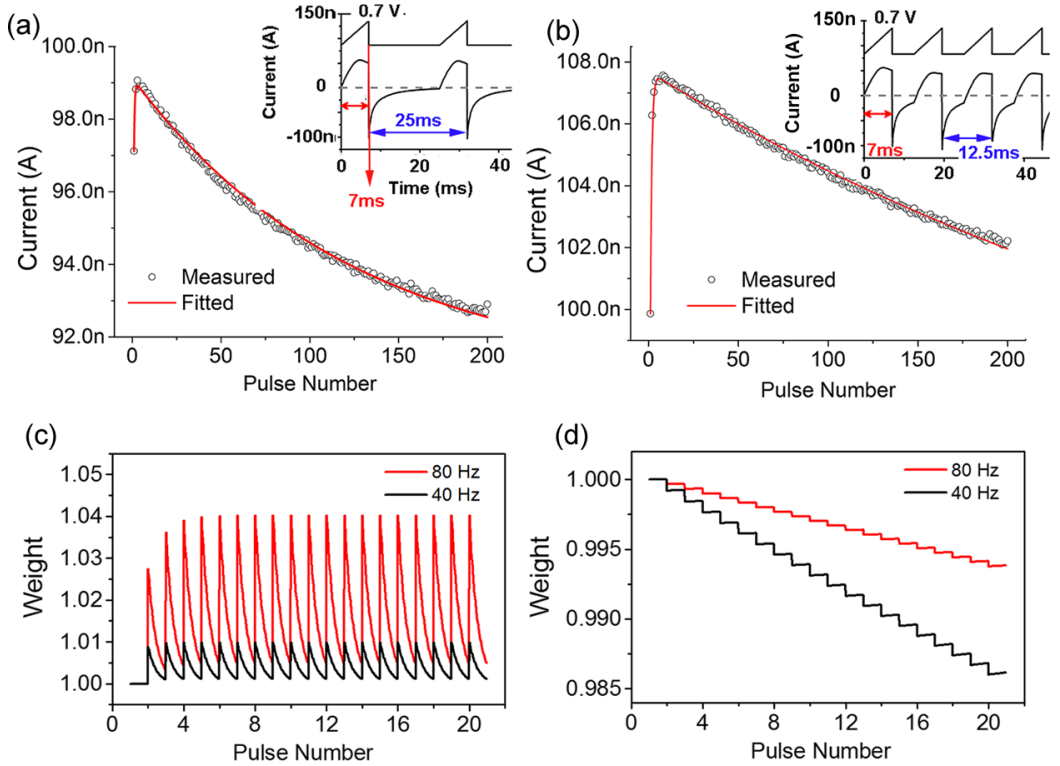

Figure 4. Pulse responses of (a) $40 \mathrm{~Hz}$ test and (b) $80 \mathrm{~Hz}$ test, in which the red lines represent the simulated results. The parameters can be found in Table S1. The time constant for both simulations are fixed: $\tau_{F}=0.0115$ and $\tau_{D}=2.2632$. Insets: Wave shapes of applied pulses and response. (c,d) Simulated value of components (c) $F$ and (d) $D$ during the first 20 pulses. The red lines and black lines represent 80 and $40 \mathrm{~Hz}$ test, respectively. The weight results were divided by the value of the first pulse. The parameters used in the simulation are the same as those in Figure $4 \mathrm{a}, \mathrm{b}$.

$$
\begin{aligned}
D_{N}= & 1-d \frac{1-b^{N}}{1-b} \\
A_{N}= & \left(1+f \frac{1-a^{N}}{1-a}\right)\left(1-d \frac{1-b^{N}}{1-b}\right) \\
W_{N}= & \left(1+f \frac{1-a^{N}}{1-a}\right) \cdot\left(1-d \frac{1-b^{N}}{1-b}\right) \\
& /[(1+f) \cdot(1-d)]
\end{aligned}
$$

All of the pulse response data were fitted using eq 14 . The fitted curves and the values of the parameters $\left(\tau_{F}, \tau_{D}, \alpha, \beta, f\right.$, and $\left.d\right)$ for each experiment can be seen in Figure S3 and Table S1. For all simulations, the values of $\tau_{F}, \tau_{D}, \alpha$, and $\beta$ are fixed at $\tau_{F}=$ $0.0115, \alpha=0.5159, \tau_{D}=2.2632$, and $\beta=1$. Figure 4a,b shows the fitting results for the pulse response to stimulations at 40 and $80 \mathrm{~Hz}$, respectively. Figure $4 \mathrm{c}$ shows the simulated values of $F$ and $D$ during the first 20 pulses for the stimulus trains of 40 and $80 \mathrm{~Hz}$ using the proposed model. $F$ and $D$ increased under bias and then exponentially decreased to 1 at intervals with their respective time constants. In both stimulations, $F$ reached a steady state very fast, indicating that the accumulation of $\mathrm{Ca}^{2+}$ ions is nearly saturated after the first few pulses, whereas the depression period, that is, the doping process was much longer. Under $80 \mathrm{~Hz}$ stimulations, the value of $F$ is larger, and the value of $D$ is smaller than that under $40 \mathrm{~Hz}$ stimulations, which is consistent with the weight dependence of frequency, as a result of the frequency selectivity.

In biological synapses, facilitation is derived from an increase in the release probability, caused by specific binding between the residual presynaptic calcium and the release sites. On the other hand, depression is the consequence of transient deactivation of the release sites after being occupied by a calcium-bound molecule. In our double-layer device, an increase in the $\mathrm{Ca}^{2+}$ ion accumulation at the $\mathrm{PEO} / \mathrm{P} 3 \mathrm{HT}$ interface can take the role of residual presynaptic calcium and determine the magnitude of the facilitation and depression components. This intrinsic property, similar to real synapses, provides a possibility to mimic synaptic plasticity by manipulating the internal molecular dynamics. Besides, the associated components $F$ and $D$ can be treated as multiple state variables, which is a concept also utilized in second-order memristors to realize several synaptic learning rules. ${ }^{41}$

The combination of $F$ and $D$ in a single device also contributes to the frequency selectivity of the device. As shown in Figure $2 \mathrm{~b}$, the device exhibits an inhibitory property $(W<1)$ under LFS (less than $80 \mathrm{~Hz}$ ) and an excitatory property under HFS $(80-125 \mathrm{~Hz})$. The origin of this phenomenon is related to the great disparity between the magnitudes of $\tau_{F}$ and $\tau_{D}$, making the $F$ and $D$ components exhibit different sensibilities to frequency. These results show that the above model naturally leads to frequency selectivity abilities in both biological synapses and in our device, which endows our device with the biolike filter effect for signal processing of neuromorphic chips. $^{38}$

2.2. Weight Modification of Post-Tetanic Stimulation. Because the magnitudes of the time constants of $F$ and $D$ differ greatly, it is natural to engineer the intervals of external pulses to find out more biolike behaviors. Here, we use the posttetanic stimulation of biological experiments to demonstrate the memory effect in our device. ${ }^{16}$ Figure 5a shows the simulated responses in two kinds of biosynapses and the corresponding synaptic weights during and following HFS, as accomplished by Zucker and Regehr. ${ }^{16}$ First, an input at $0.5 \mathrm{~Hz}$ was loaded, where the amplitude of the PSC remained unchanged. Then, the synapses were stimulated by a train of high-frequency pulses $(10 \mathrm{~Hz}, 10 \mathrm{~s})$, the so-called tetanic stimulation, where the weight changed bidirectionally based on the type of facilitation or depression present. When returning to LFS, the plasticity of facilitation vanished immediately, and the depression effect persisted for a couple of seconds before gradually fading away. The change in synapse activity during a train of stimuli can 
(a)

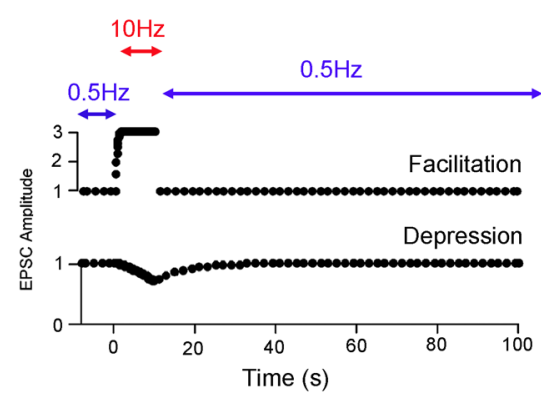

(c)

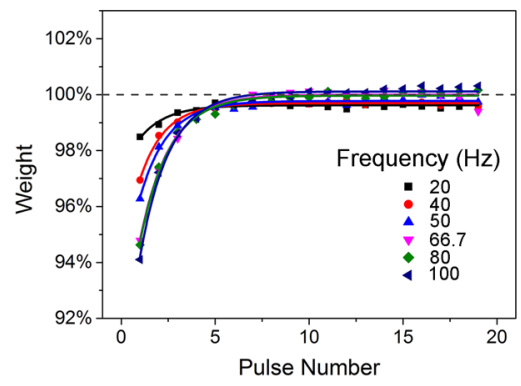

(b)

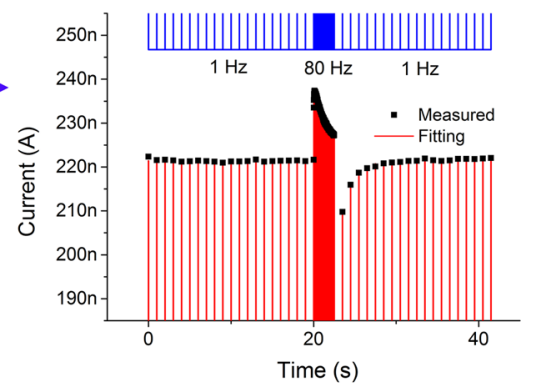

(d)

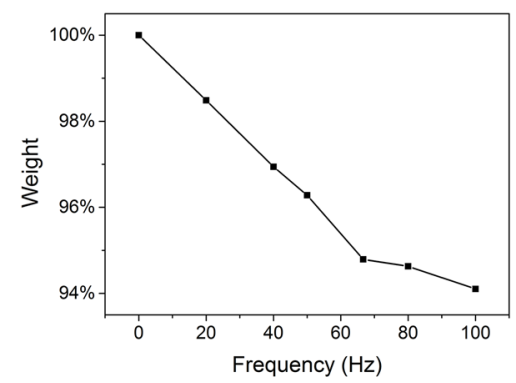

Figure 5. (a) Simulations of the responses to post-tetanic stimulation in facilitation synapse and depression synapse. ${ }^{16}$ The pulse frequencies used are $0.5,10$, and $0.5 \mathrm{~Hz}$. (b) Pulse responses to a set of stimulations with frequency series of $1 \mathrm{~Hz} \rightarrow$ tetanic stimulations $(80 \mathrm{~Hz}) \rightarrow 1 \mathrm{~Hz}$. The pulse numbers of the three sections are 20,200, and 20 . We assumed that the response of $1 \mathrm{~Hz}$ stimulation before the tetanus stimulus keeps constant. The response of $80 \mathrm{~Hz}$ stimulation was fitted by the model, and the $1 \mathrm{~Hz}$ stimulation was fitted using parameters deduced from the data of the tetanus stage. (c) Post-tetanic plasticity under different frequencies of tetanic stimulation. The measured and fitted data are shown as dots and lines, respectively. (d) Weight of the first PSC during LFS after the tetanus stimulations at different frequencies.

remain for a long time, which is an important characteristic that allows synapses to have the ability to learn and have memory.

The total post-tetanic plasticity of a realistic synapse is usually the weighted combination of these several kinds. We stimulated our device by using a complex train of pulses (triangular pulses with an amplitude of $0.7 \mathrm{~V}$ and a loading rate of $100 \mathrm{~V} / \mathrm{s}$ ) consisting of $1 \mathrm{~Hz}$ (20 pulses)-high frequency (200 pulses) $-1 \mathrm{~Hz}$ (20 pulses), to emulate pretetanic, tetanic, and post-tetanic stimulations, respectively. Figure $5 \mathrm{~b}$ shows the response current to these stimuli. During pretetanic stimulation, the synaptic weight remained almost constant. When the tetanic stimulation was started, the device exhibited fast facilitation and slow depression. After HFS, the weight decayed to a depression state with a time constant of several seconds, which is the result of the combination of a facilitationdominated synapse and a depression-dominated synapse, as shown in Figure 5a.

We fitted the measured data presented in Figure $5 b$ by using the previously discussed model. When stimulations change from high frequency to $1 \mathrm{~Hz}, F$ quickly decays to 1 because $\tau_{F}$ $\ll 1$. Thus, the post-tetanic synaptic weight mainly depends on $D$ as

$$
\begin{aligned}
& D_{N}=1-\left(1-D_{N-1}\right) \cdot b-d \\
& b=\beta \cdot \exp \left(-\frac{\Delta t}{\tau_{D}}\right), \quad \Delta t=1 \mathrm{~s}
\end{aligned}
$$

The initial value used for $D$ is $D_{200}$, which corresponds to the last PSC of the high-frequency stimulus. The general term then turns into

$$
\begin{aligned}
& P_{N}=\left(D_{200}-p \frac{1-d^{N}}{1-d}\right) /(1-d) \\
& p=D_{200}-D_{201}, \quad p<0
\end{aligned}
$$

where $N$ is the number of post-tetanic pulses. The fitted curves are shown in Figure 4c. We assume that the pretetanic stimulation does not change the synaptic weight; so, the plasticity during tetanus is suitable for the model previously discussed. The fitting parameters are shown in Table S2.

The fitting results show that the intrinsic ionic kinetics substantially contributes to synaptic plasticity on different time scales. Early studies indicated that when the frequency changed, the pulse response of an artificial synapse would change accordingly, having a stable weight for each stage. ${ }^{42,43}$ Therefore, when the total plasticity at a high frequency was in a facilitation state, the synaptic weight after tetanus discontinuously jumped to a depression state. This characteristic, based on multistate variables with different time constants, is worth considering because it provides an innovative way of manipulating synaptic plasticity, emulating filtering, or memorizing.

\section{CONCLUSIONS}

In summary, $\mathrm{Pt} / \mathrm{Ca}^{2+}-\mathrm{PEO} / \mathrm{P} 3 \mathrm{HT} / \mathrm{Pt}$ devices responded to pulse stimulations analogously to biological SC synapses. The PSC value first increased and then descended with increasing input number in a pulse train. The weight of the PSC decreased at LFS but increased at HFS. However, the NDR peak during the charging process exhibited the opposite trend. These behaviors suggest that our device has the ability to transfer signals bidirectionally, confirming the equivalence between the ionic kinetics of our device and the transmitter kinetics of the 
SC synapse. We adopted a facilitation-depression interplay model, which corresponds to the ionic polarization and doping interplay at the electrolyte/semiconducting polymer interface, to successfully mimic the weight modification of the PSC of our device. We verified that the great disparity observed between the recovery time constants of the two components $\left(\tau_{F}\right.$ and $\left.\tau_{D}\right)$ accounted for the synaptic plasticity. Moreover, we demonstrated that such an interplay was able to emulate the features of the response to post-tetanic stimulation, as well as that observed in neuroscience. Therefore, our study shows a great opportunity regarding the combination of the physical basis of biological synapses and the intrinsic ionic kinetics of artificial devices, enabling a new method to simulate synapses for more efficient neuromorphic computing.

\section{EXPERIMENTAL SECTION}

The conjugated polymers P3HT $\left(M_{\mathrm{W}}=65000 \mathrm{~g} \mathrm{~mol}^{-1}\right.$, Beijing Sweet Technology Co., Ltd.), PEO $\left(M_{\mathrm{W}}=100000 \mathrm{~g} \mathrm{~mol}^{-1}\right.$, Sigma-Aldrich Co. Ltd.), and calcium trifluoromethane sulfonate $\left[\mathrm{Ca}\left(\mathrm{CF}_{3} \mathrm{SO}_{3}\right)_{2}, 98 \%\right.$, Sigma-Aldrich Co. Ltd. $]$ were used as purchased. $\mathrm{P} 3 \mathrm{HT}$ was dissolved at a concentration of 5 $\mathrm{mg} \mathrm{mL}^{-1}$ in 2-dichlorobenzene. PEO was dissolved at a concentration of $5 \mathrm{mg} \mathrm{mL} \mathrm{m}^{-1}$ in deionized water containing $\mathrm{Ca}\left(\mathrm{CF}_{3} \mathrm{SO}_{3}\right)_{2}$ at different molar ratios of $1: 32$ and zero, expressed as the ratio of $\mathrm{Ca}\left(\mathrm{CF}_{3} \mathrm{SO}_{3}\right)_{2}$ to the ethylene oxide (EO) monomer. The solvents were fully dispersed by stirring on a magnetic hot plate at $320 \mathrm{~K}$ for $12 \mathrm{~h}$. Silicon substrates (1 $\times 1 \mathrm{~cm}^{2}$ ) with a layer of Pt of thickness $100 \mathrm{~nm}$ were used as the bottom electrode and cleaned in an ultrasonic bath with acetone, alcohol, and deionized water in sequence.

The substrates were spin-coated with $2 \mu \mathrm{L}$ of the P3HT solution at 500, 3000, and $1500 \mathrm{rpm}$ for 10,30 , and $20 \mathrm{~s}$, respectively. They were subsequently dried on a hot plate, first at a temperature of $373 \mathrm{~K}\left(100{ }^{\circ} \mathrm{C}\right)$ for $1 \mathrm{~h}$ and then at a temperature of $393 \mathrm{~K}\left(120^{\circ} \mathrm{C}\right)$ for $20 \mathrm{~min}$. Next, $5 \mu \mathrm{L}$ of the $\mathrm{PEO}-\mathrm{Ca}\left(\mathrm{CF}_{3} \mathrm{SO}_{3}\right)_{2}$ solution was drop-cast on the P3HT film and baked at $383 \mathrm{~K}$ for $20 \mathrm{~min}$. The preparation of the films was completed in a glove box filled with nitrogen. Finally, 70 nm-thick Pt island electrodes with a diameter of $100 \mu \mathrm{m}$ were deposited on top of the films using electron beam evaporation.

Electrical measurements were carried out on a semiconductor device measurement platform (Agilent B1500A) containing an arbitrary function generator (Agilent B1530). The electrical signal was captured using probes placed on the top and bottom electrodes. Scanning electron microscopy (SEM) images were obtained using a ZEISS EVO scanning electron microscope. Raman spectra were obtained using an HR-800 Raman system with a $633 \mathrm{~nm} \mathrm{HeNe} \mathrm{laser} \mathrm{and} \mathrm{a}$ resolution of $1 \mathrm{~cm}^{-1}$. XRD patterns for salt-doped PEO were obtained using a Rigaku Smartlab apparatus with a resolution of $1^{\circ} / \mathrm{min}$ and a scanning range of $2 \theta=15-25^{\circ}$. Finally, polarizing microscope images were observed using a Leica DM2500 M polarizing microscope. XRD patterns for P3HT films were obtained using a D8 Advance diffractometer under $\theta-2 \theta$ out-of-plain scanning. The increment is $0.01^{\circ}$, and the scanning speed is $0.03^{\circ}$ step. The result was collected for $2 \theta$ ranging from 4 to $10^{\circ}$.

\section{ASSOCIATED CONTENT}

\section{S Supporting Information}

The Supporting Information is available free of charge on the ACS Publications website at DOI: 10.1021/acsomega.6b00392.
Structure of PEO, P3HT and $\mathrm{PEO} / \mathrm{P} 3 \mathrm{HT}$, electrical properties of $\mathrm{PEO}-\mathrm{Ca}\left(\mathrm{CF}_{3} \mathrm{SO}_{3}\right)_{2}$ single layer, $\mathrm{P} 3 \mathrm{HT}$ single layer, and $\mathrm{PEO} / \mathrm{P} 3 \mathrm{HT}$ double layer, pulse responses of the $\mathrm{Ca}^{2+}-\mathrm{PEO}$ single-layer device, fit curves and the values of the parameters for the pulse responses of the PEO-Ca $\left(\mathrm{CF}_{3} \mathrm{SO}_{3}\right)_{2} / \mathrm{P} 3 \mathrm{HT}$ double-layer device, and simulation parameters for the post-tetanic stimulation experiment (PDF)

\section{AUTHOR INFORMATION}

\section{Corresponding Authors}

*E-mail: zengfei@mail.tsinghua.edu.cn (F.Z.).

*E-mail: panf@mail.tsinghua.edu.cn (F.P.).

ORCID

Fei Zeng: 0000-0001-8735-8766

\section{Author Contributions}

Y.H. performed all experiments and wrote the paper. F.Z. designed the experiments and wrote the paper. All authors have given approval to the final version of the manuscript. C.C. contributed to the construction of the model, W.D. contributed to sample preparation, and X.L. contributed to electrical analysis. F.P. discussed the work.

\section{Notes}

The authors declare no competing financial interest.

\section{ACKNOWLEDGMENTS}

Our research was supported by the National Natural Science foundation of China (grant nos. 51371103 and 51231004), Brain Inspired Computing Research of Tsinghua University (20141080934), and the Opening Project of Key Laboratory of Microelectronic Devices \& Integrated Technology, Institute of Microelectronics, Chinese Academy of Sciences.

\section{ABBREVIATIONS}

P3HT, poly[3-hexylthiophene-2,5-diyl]; PEO, polyethylene oxide; STP, short-term plasticity; NDR, negative differential resistance; PSC, post-synaptic current; LFS, low frequency stimulation; HFS, high frequency stimulation; SRDP, spikerate-dependent plasticity; SC, Schaffer collateral

\section{REFERENCES}

(1) Tuma, T.; Pantazi, A.; Le Gallo, M.; Sebastian, A.; Eleftheriou, E. Stochastic phase-change neurons. Nat. Nanotechnol. 2016, 11, 693699.

(2) Jo, S. H.; Chang, T.; Ebong, I.; Bhadviya, B. B.; Mazumder, P.; $\mathrm{Lu}, \mathrm{W}$. Nanoscale memristor device as synapse in neuromorphic systems. Nano Lett. 2010, 10, 1297-1301.

(3) Zhu, L. Q.; Wan, C. J.; Guo, L. Q.; Shi, Y.; Wan, Q. Artificial synapse network on inorganic proton conductor for neuromorphic systems. Nat. Commun. 2014, 5, 3158.

(4) Pickett, M. D.; Medeiros-Ribeiro, G.; Williams, R. S. A scalable neuristor built with Mott memristors. Nat. Mater. 2013, 12, 114-117.

(5) Adamatzky, A.; Chua, L. Memristor Networks, 1 ed.; Springer: Switzerland, 2014.

(6) Shashoua, V. E. Electrically Active Polyelectrolyte Membranes. Nature 1967, 215, 846-847.

(7) Heimburg, T.; Jackson, A. D. On soliton propagation in biomembranes and nerves. Proc. Natl. Acad. Sci. U.S.A. 2005, 102, 9790-9795.

(8) Zeng, F.; Lu, S.; Li, S.; Li, X.; Pan, F. Frequency Selectivity in Pulse Responses of Pt/Poly(3-hexylthiophene-2,5-diyl)/Polyethylene Oxide $+\mathrm{Li}^{+} /$Pt Hetero-Junction. PLoS One 2014, 9, No. e108316. 
(9) Dong, W. S.; Zeng, F.; Lu, S. H.; Li, X. J.; Chang, C. T.; Liu, A.; Pan, F.; Guo, D. Effect of heavy-ion on frequency selectivity of semiconducting polymer/electrolyte heterojunction. RSC Adv. 2015, 5, 98110-98117.

(10) Dong, W. S.; Zeng, F.; Lu, S. H.; Liu, A.; Li, X. J.; Pan, F. Frequency-dependent learning achieved using semiconducting polymer/electrolyte composite cells. Nanoscale 2015, 7, 16880-16889.

(11) Dong, W.; Zeng, F.; Hu, Y.; Chang, C.; Li, X.; Pan, F.; Li, G. Sliding Threshold of Spike-Rate Dependent Plasticity of a Semiconducting Polymer/Electrolyte Cell. J. Polym. Sci., Part B: Polym. Phys. 2016, 54, 2412-2417.

(12) Cooper, L. N.; Bear, M. F. The BCM theory of synapse modification at 30: Interaction of theory with experiment. Nat. Rev. Neurosci. 2012, 13, 798-810.

(13) Dudek, S. M.; Bear, M. F. Homosynaptic long-term depression in area CAl of hippocampus and effects of $N$-methyl-D-aspartate receptor blockade. Proc. Natl. Acad. Sci. U.S.A. 1992, 89, 4363-4367.

(14) Chang, C. T.; Zeng, F.; Li, X. J.; Dong, W. S.; Lu, S. H.; Gao, S.; Pan, F. Simulation of synaptic short-term plasticity using Ba$\left(\mathrm{CF}_{3} \mathrm{SO}_{3}\right)_{2}$-doped polyethylene oxide electrolyte film. Sci. Rep. 2016, 6,18915 .

(15) Abbott, L. F.; Regehr, W. G. Synaptic computation. Nature 2004, 431, 796.

(16) Zucker, R. S.; Regehr, W. G. Short-term synaptic plasticity. Annu. Rev. Physiol. 2002, 64, 355-405.

(17) Dittman, J. S.; Kreitzer, A. C.; Regehr, W. G. Interplay between facilitation, depression, and residual calcium at three presynaptic terminals. J. Neurosci. 2000, 20, 1374-1385.

(18) Jiang, X.; Shen, S.; Cadwell, C. R.; Berens, P.; Sinz, F.; Ecker, A. S.; Patel, S.; Tolias, A. S. Principles of connectivity among morphologically defined cell types in adult neocortex. Science 2015, $350,9462$.

(19) Bear, M. F.; Connors, B. W.; Paradiso, M. A. Neuroscience: Exploring the Brain; High Education Press: Beijing, 2007.

(20) Regehr, W. G. Short-term presynaptic plasticity. Cold Spring Harbor Perspect. Biol. 2012, 4, a005702.

(21) Dittman, J. S.; Regehr, W. G. Calcium dependence and recovery kinetics of presynaptic depression at the climbing fiber to Purkinje cell synapse. J. Neurosci. 1998, 18, 6147-6162.

(22) Liu, A.; Zeng, F.; Hu, Y.; Lu, S.; Dong, W.; Li, X.; Chang, C.; Guo, D. Simulation of pulse responses of lithium salt-doped poly ethyleneoxide. J. Polym. Sci., Part B: Polym. Phys. 2016, 54, 831-837.

(23) Panzer, M. J.; Frisbie, C. D. Polymer electrolyte-gated organic field-effect transistors: Low-voltage, high-current switches for organic electronics and testbeds for probing electrical transport at high charge carrier density. J. Am. Chem. Soc. 2007, 129, 6599.

(24) Brody, D. L.; Yue, D. T. Release-Independent Short-Term Synaptic Depression in Cultured Hippocampal Neurons. J. Neurosci. 2000, 20, 2480-2494.

(25) Lu, S.; Zeng, F.; Dong, W.; Liu, A.; Li, X.; Luo, J. Controlling Ion Conductance and Channels to Achieve Synaptic-like Frequency Selectivity. Nano-Micro Lett. 2015, 7, 121-126.

(26) Zeng, F.; Lu, S. H.; Dong, W. S.; Liu, A.; Li, X. J.; Chang, C. T. Thresholds of frequency selectivity of $\mathrm{Pt} / \mathrm{poly}$ (3-hexylthiophene-2,5diyl)/polyethylene oxide $+\mathrm{Mg}^{2+} / \mathrm{Pt}$ heterojunctions. Solid State Ionics 2016, 287, 42-47.

(27) Cooper, L. N.; Liberman, F.; Oja, E. A theory for the acquisition and loss of neuron specificity in visual cortex. Biol. Cybern. 1979, 33, 9-28.

(28) Li, S.; Zeng, F.; Chen, C.; Liu, H.; Tang, G.; Gao, S.; Song, C.; Lin, Y.; Pan, F.; Guo, D. Synaptic plasticity and learning behaviours mimicked through $\mathrm{Ag}$ interface movement in an $\mathrm{Ag} /$ conducting polymer/Ta memristive system. J. Mater. Chem. C 2013, 1, 52925298.

(29) Zeng, F.; Li, S.; Yang, J.; Pan, F.; Guo, D. Learning processes modulated by the interface effects in a $\mathrm{Ti} /$ conducting polymer/ $\mathrm{Ti}$ resistive switching cell. RSC Adv. 2014, 4, 14822.

(30) Wang, Z. Q.; Xu, H. Y.; Li, X. H.; Yu, H.; Liu, Y. C.; Zhu, X. J. Synaptic learning and memory functions achieved using oxygen ion migration/diffusion in an amorphous InGaZnO memristor. Adv. Funct. Mater. 2012, 22, 2759-2765.

(31) Yang, X.; Wang, C.; Shang, J.; Zhang, C.; Tan, H.; Yi, X.; Pan, L.; Zhang, W.; Fan, F.; Liu, Y.; Chen, Y.; Liu, G.; Li, R.-W. An organic terpyridyl-iron polymer based memristor for synaptic plasticity and learning behavior simulation. RSC Adv. 2016, 6, 25179-25184.

(32) Li, Y.; Zhong, Y.; Xu, L.; Zhang, J.; Xu, X.; Sun, H.; Miao, X. Ultrafast Synaptic Events in a Chalcogenide Memristor. Sci. Rep. 2013, 3, 1619.

(33) Li, Y.; Zhong, Y.; Zhang, J.; Xu, L.; Wang, Q.; Sun, H.; Tong, $\mathrm{H}$.; Cheng, X.; Miao, X. Activity-dependent synaptic plasticity of a chalcogenide electronic synapse for neuromorphic systems. Sci. Rep. 2014, 4, 4906.

(34) Hebb, D. O. The Organization of Behavior: A Neuropsychological Theory; Wiley, 1949.

(35) Spruston, N.; Kath, W. L. Dendritic arithmetic. Nat. Neurosci. 2004, 7, 567-569.

(36) Panzer, M. J.; Frisbie, C. D. Polymer electrolyte-gated organic field-effect transistors: Low-voltage, high-current switches for organic electronics and testbeds for probing electrical transport at high charge carrier density. J. Am. Chem. Soc. 2007, 129, 6599.

(37) Kaake, L. G.; Zhu, X.-Y. Charge Transport, Nanostructure, and the Mott Insulator-to-Metal Transition in Poly(3-hexylthiophene). J. Phys. Chem. C 2008, 112, 16174-16177.

(38) Varela, J. A.; Sen, K.; Gibson, J.; Fost, J.; Abbott, L. F.; Nelson, S. B. A quantitative description of short-term plasticity at excitatory synapses in layer $2 / 3$ of rat primary visual cortex. J. Neurosci. 1997, 17, 7926.

(39) Lisman, J. Bursts as a unit of neural information: Making unreliable synapses reliable. Trends Neurosci. 1997, 20, 38-43.

(40) Zador, A. M.; Dobrunz, L. E. Dynamic synapses in the cortex. Neuron 1997, 19, $1-4$.

(41) Bi, G. Q.; Poo, M. M. Synaptic modifications in cultured hippocampal neurons: Dependence on spike timing, synaptic strength, and postsynaptic cell type. J. Neurosci. 1998, 18, 10464-10472.

(42) Du, C.; Ma, W.; Chang, T.; Sheridan, P.; Lu, W. D. Biorealistic Implementation of Synaptic Functions with Oxide Memristors through Internal Ionic Dynamics. Adv. Funct. Mater. 2015, 25, 4290-4299.

(43) Alibart, F.; Pleutin, S.; Guérin, D.; Novembre, C.; Lenfant, S.; Lmimouni, K.; Gamrat, C.; Vuillaume, D. An organic nanoparticle transistor behaving as a biological spiking synapse. Adv. Funct. Mater. 2010, 20, 330-337. 